\title{
DISTÚRBIOS FÍSICOS E COMPORTAMENTAIS EM CÃES E GATOS IDOSOS
}

\section{(Physical and behavioral disturbance in aging dogs and cats)}

\author{
FREITAS, E.P. ${ }^{1}$; RAHAL, S.C. ${ }^{2}$; CIANI, R.B. ${ }^{3}$ \\ 1Aluna do Programa de Pós-graduação em Medicina Veterinária, Faculdade de Medicina Veterinária e \\ Zootecnia (FMVZ), Unesp Botucatu; \\ ${ }^{2}$ Departamento de Cirurgia e Anestesiologia Veterinária, FMVZ, Unesp Botucatu; \\ ${ }^{3}$ Médica Veterinária, Clinivet - Jaú.
}

\begin{abstract}
RESUMO - A senescência é um processo que se estende do nascimento até a morte e refere-se às disfunções que ocorrem com o avançar da idade. O trabalho teve por objetivo realizar um levantamento das principais alterações físicas e comportamentais, bem como o estabelecimento de correlação entre peso corpóreo e o tipo de alimentação, em 63 cães e 27 gatos,com idade acima de oito anos. Os resultados permitiram concluir que o distúrbio físico de maior ocorrência foi a obesidade. Dentre as mudanças comportamentais, a ansiedade e a agressividade foram as mais prevalentes. A combinação de comida caseira com ração comercial constituiu na principal fonte de alimentação oferecida pelos proprietários, sendo a mais consumida pelos animais obesos.
\end{abstract}

Palavras-chave: idoso; alimento; comportamento; cães; gatos.

\begin{abstract}
Senescence is a process that extends from birth until death, and refers to the dysfunction that occurs with aging. The aim of this study is to make a survey of the main physical and behavioral changes, as well as to establish the correlation between the body weight and type of food in 63 dogs and 27 cats over 8 years old. It was possible to conclude that the obesity was the most frequent physical disturbance. Among the behavioral changes, anxiety and aggressiveness were the most prevalent. The commercial pet food combined to home made diet was the principal type of food provided by the owners, and this food was the most ingested by the obese animals.
\end{abstract}

Key-words: aging; food; behavior; dogs; cats.

\section{Introdução}

O envelhecimento é um processo que se estende do nascimento até a morte (SHEFFY e WILLIAMS, 1981). Embora ocorram muitas variações individuais entre animais, cães de raças grandes e gigantes são considerados geriátricos aos cinco anos de idade, ao passo que os gatos, bem como os cães de raças pequenas ou médias, são estimados como de meia idade até os sete anos (MARKHAM e HODGKINS, 1989). Cães que apresentaram uma média de vida mais longa faleceram em virtude de doenças apropriadas à idade, em especial câncer (BRONSON, 1982; TAYLOR et al., 1995; MALINOWSKI, 2006).

Um animal sênior se torna menos ativo. Os níveis de lipídio, colesterol e fosfolipídio tendem a aumentar (JEUSETTE et al., 2005), assim como a porcentagem de peso em virtude do aumento da gordura corpórea e sua distribuição, devido à inabilidade de metabolizar lipídeos (SHEFFY e WILLIAMS, 1981).

As doenças cardíacas são causa comum de morbidade e mortalidade em cães idosos (MARKHAM e HODGKINS, 1989), sendo que $30 \%$ sofrem de endocardiose (TAYLOR et al., 1995). Em gatos, o aparelho respiratório é mais susceptível a doenças pulmonares e bronquite crônica (MOSIER, 1989). Além disso, gatos idosos podem ter constipação ou diarréia. Quando não há resposta a medicamentos ou mudanças alimentares, a constante evacuação pode estar associada a lesões renais, hepáticas ou pancreáticas; estresse nessa espécie pode induzir crise urêmica aguda, podendo ser fatal a despeito do cuidado intensivo (CARE, 1983).

Com o envelhecimento também ocorre o declínio da secreção dos hormônios produzidos pelas gônadas. Os testículos desenvolvem atrofia flácida e estão propensos à formação de tumores. Os ovários apresentam aumento gradual de peso e posteriormente atrofiam. As glândulas mamárias mostram evidência de doença fibrocística ou neoplásica (MOSIER,1989).

Entre os problemas comportamentais em animais idosos estão a ansiedade da separação, agressão às pessoas e entre as espécies, vocalização excessiva, controle inadequado de necessidades fisiológicas, fobias a barulho, compulsão e dificuldade para dormir à noite (LANDSBERG e ARAUJO, 2005). Os distúrbios sociais podem estar associados à morte de um animal contactante ou à introdução de outro animal de estimação (HOUPT e BEAVER, 1981).

Por meio de entrevista telefônica com os proprietários foram avaliadas 97 cadelas e 83 cães machos, castrados, com idade variando de 11 até 16 anos. 0 aumento do enfraquecimento cognitivo, em apenas uma categoria, foi detectado em $28 \%$ de cães entre 11 e 12 anos e $68 \%$ de 15 até 16 anos (NEILSON et al., 2001). Vale ressaltar que após o diagnóstico do problema comportamental e a determinação da causa, o processo pode ser controlado ou corrigido com modificações ambientais ou terapias com drogas (LANDSBERG e 
ARAUJO, 2005).

Com relação aos aspectos nutricionais, a dieta é fator determinante para a condição corpórea dos animais. A obesidade é um dos mais prevalentes distúrbios de cães e gatos idosos, sendo que $44 \%$ dos cães apresentam excesso de peso, e pode estar relacionada ou a uma quantidade excessiva de alimento fornecido ou a uma utilização inadequada de energia (GERMAN, 2006). A diminuição da quantidade de comida oferecida não é a maneira mais adequada para obter a perda de peso; a redução total de energia é a prática mais segura (TAYLOR et al., 1995). Foi avaliada em cães, por um período de dois anos, a resposta de quatro dietas contendo porcentagens variadas de proteína e energia. A habilidade de cães senis em utilizar nutrientes não foi significativamente diferente de adultos jovens (SHEFFY et al., 1985).

Em virtude do exposto, este estudo tem por objetivo analisar dados retrospectivos colhidos de cães e gatos idosos, examinados em uma clínica veterinária particular, enfatizando as alterações físicas e comportamentais conseqüentes à senescência, e estabelecer uma correlação entre peso corpóreo e o tipo de alimento oferecido.

\section{Material e Métodos}

Efetuou-se análise retrospectiva de três anos com relação a cães e gatos senis que foram atendidos em uma clínica veterinária particular, localizada na cidade de Jaú, interior de São Paulo.

Foi desenvolvido um questionário e, por meio de entrevista telefônica, foram colhidos os seguintes dados: espécie, raça, idade, sexo (castrado ou inteiro), tipo de alimentação (ração comercial ou comida caseira), padrão corporal, ganho de peso após castração e motivo do atendimento clínico. Também foram abordadas questões de aspecto comportamental como alterações ocorridas com o avançar da idade, orientação dentro de casa (se o animal esbarrava em objetos), interação social e problemas na ausência do dono (ansiedade, agressividade com membros da família e com outros animais, eliminação de dejetos pela casa), ciclo do sono (dificuldade para dormir à noite) e vocalização inapropriada ou excessiva.

Os dados obtidos foram submetidos ao teste Exato de Fisher bilateral $(\mathrm{Pr}<=\mathrm{P})$, utilizando-se o programa computacional SAS. Os resultados foram obtidos no nível de $5 \%$ de significância.

\section{Resultados e Discussão}

Foram estudados 90 animais, com idade entre oito e 17 anos. Deste total, havia 63 cães, sendo 23 machos e 40 fêmeas, e 27 gatos, sendo sete machos e 20 fêmeas (FIGURA 1). A faixa etária predominante dos gatos foi de 10 anos $(40,74 \%)$ e a idade máxima de 14 anos (3,70\%). Para os cães, o maior número de animais situou-se entre nove e 10 anos (42,86\%), sendo a idade máxima de 17 anos (1,59\%). Isso comprova um crescente aumento na longevidade, tanto para cães quanto gatos, fato resultante dos avanços científicos e tecnológicos no diagnóstico e tratamento das doenças, bem como na área da nutrição animal (PUGLIESE et al., 2005).

FIGURA 1 - DISTRIBUIÇÃO DOS 90 ANIMAIS ESTUDADOS, DE ACORDO COM O SEXO E A ESPÉCIE.

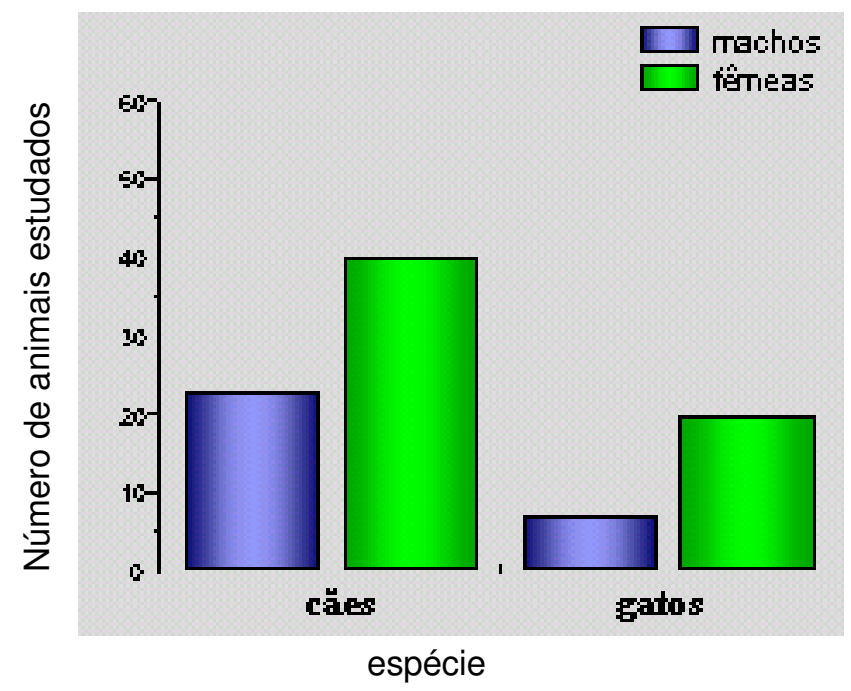


Foram considerados obesos pelos proprietários 41 $(45,56 \%)$ dos 90 animais, sendo 31 (75,60\%) caninos (20 fêmeas e 11 machos) e 10 (24,40\%) felinos (sete fêmeas e três machos), comprovando a maior propensão do ganho de peso na senescência (TAYLOR et al., 1995), que pode estar associada à inabilidade de metabolizar lipídeos (SHEFFY e WILLIAMS, 1981) e atividade física reduzida (MARKHAM e HODGKINS, 1989).

Quanto à alimentação, dos 63 cães, 11 (17,46\%) ingeriam ração comercial, 15 (23,80\%) recebiam comida caseira e 37 (58,73\%) eram alimentados com ração comercial mais comida caseira. Para os 27 gatos verificou-se que oito $(29,63 \%)$ recebiam ração comercial e $19(70,37 \%)$ ração comercial mais comida caseira.
Correlacionando os animais obesos com o tipo de alimentação fornecida (FIGURA 2), denotou-se que, dentre as cadelas, $14(45,16 \%)$ ingeriam ração comercial e comida caseira, quatro (12,90\%) comida caseira e duas $(6,45 \%)$ ração. Dentre os cães machos, cinco $(16,13 \%)$ recebiam ração mais comida caseira, três $(9,68 \%)$ comida caseira e três $(9,68)$ ração comercial. Para os felinos foi constatado que, entre as fêmeas, quatro (40\%) eram alimentadas com ração comercial e três (30\%) com ração e comida caseira; já entre os machos, três (30\%) ingeriam ração mais comida caseira. A combinação dos dois tipos de alimento favorece a ingestão excessiva de energia, um fator contribuinte para a obesidade (GERMAN, 2006).

\section{FIGURA 2 - CORRELAÇÃO ENTRE ANIMAIS OBESOS E O TIPO DE ALIMENTO OFERECIDO PELOS PROPRIETÁRIOS.}

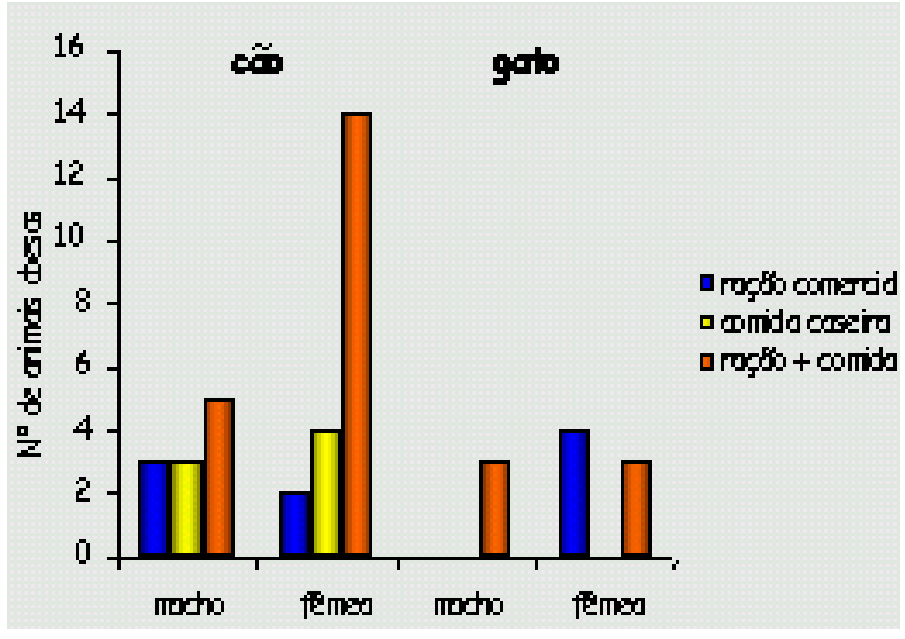

Do total de animais estudados foram obtidos 44 $(48,89 \%)$ cães inteiros e 19 (21,11\%) castrados, cujo período de intervenção cirúrgica foi muito variável. Dos gatos havia seis $(6,67 \%)$ inteiros e $21(23,33 \%)$ castrados, sendo que estes foram operados com idade inferior a três anos. Foi verificado ganho de peso após a castração em $68,42 \%$ dos cães, sendo quatro machos $(21,05 \%)$ e nove fêmeas $(47,37 \%)$, e em $52,38 \%$ dos gatos, sendo três machos $(14,28 \%)$ e oito fêmeas $(38,09 \%)$, conforme a FIGURA 3. Entretanto, estatisticamente não houve correlação entre ganho de peso e castração, fato que pode estar associado ao número baixo da amostra, visto que a obesidade é considerada uma das desvantagens da neutralização dos animais (CONCANNON, 1995).

Quanto aos problemas médicos do animal geriátrico, sabe-se que no envelhecimento ocorre redução gradual de todas as funções fisiológicas, há diminuição da competência imune e aumenta a predisposição para doenças infecciosas e neoplásicas (MARKHAM e HODGKINS, 1989; MOSIER, 1989; GREELEY et al., 2001). Dos 63 cães pesquisados, os tumores foram a principal manifestação clínica encontrada, isto é, 19,05\% $(12 / 63)$, em segundo ficaram as gastrenterites e os problemas de pele com 11,11\% (7/63) cada uma, seguida por otite $7,94 \%(5 / 63)$ e piometra $4,76 \%$ (3/ 63). Dentre os 27 felinos estudados, $18,52 \%(5 / 27)$ apresentaram problemas respiratórios, 11,11\% (3/27) manifestaram algum tipo de tumor. Ocorreu o mesmo percentual de $7,41 \%$ (2/27) à doença periodontal, piometra e feridas cutâneas. Embora o aparelho respiratório do paciente idoso seja susceptível a doenças pulmonares e bronquite crônica (MOSIER,1989), a alta prevalência de alterações respiratórias neste estudo pode eventualmente estar associada à não vacinação contra a rinotraqueíte e calicivirose felina (BARR et al., 1996). 


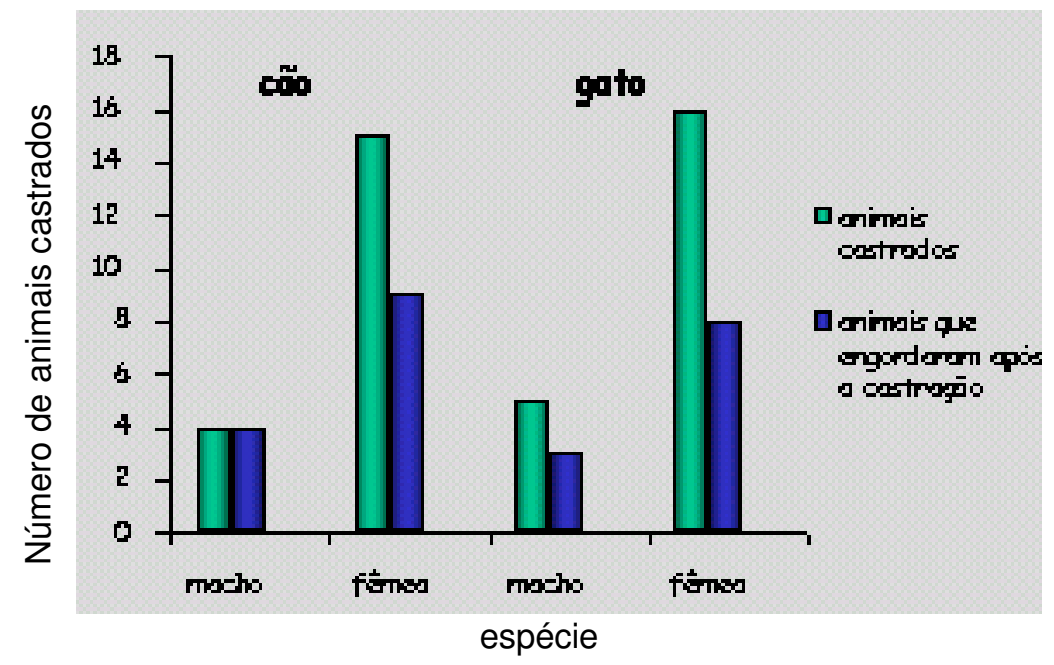

TABELA 1 - ALTERAÇÕES COMPORTAMENTAIS EM ANIMAIS IDOSOS DAS ESPÉCIES CANINA E FELINA.

\begin{tabular}{|c|c|c|c|c|}
\hline \multirow{3}{*}{$\begin{array}{l}\text { Alterao̧les de } \\
\text { comportamento }\end{array}$} & \multicolumn{4}{|c|}{ Epecizs } \\
\hline & \multicolumn{2}{|c|}{ conina } & \multicolumn{2}{|c|}{ feling } \\
\hline & madios & femeas & madhos & femeas \\
\hline Etarry on objetos & 4 & 5 & 1 & 0 \\
\hline $\begin{array}{l}\text { Ansicos quendo o das } \\
\text { mi de cons }\end{array}$ & 12 & 27 & 3 & 7 \\
\hline $\begin{array}{l}\text { Agressio om ar } \\
\text { peros de familia }\end{array}$ & 4 & 5 & 1 & 6 \\
\hline $\begin{array}{l}\text { Agrssio orm autros } \\
\text { animis }\end{array}$ & 6 & 16 & 5 & 11 \\
\hline $\begin{array}{l}\text { Uhine e defor dortro } \\
\text { de cond }\end{array}$ & 6 & 4 & 1 & 5 \\
\hline $\begin{array}{l}\text { Difiauldade para } \\
\text { domirn à noite }\end{array}$ & 2 & 1 & 2 & 1 \\
\hline 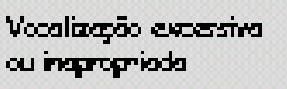 & 7 & 9 & 3 & 3 \\
\hline
\end{tabular}

Os achados referentes às alterações comportamentais nas diferentes espécies foram dispostos na TABELA 1. Os 90 animais avaliados foram divididos em dois grupos: com e sem alterações de comportamento. A proporção dos que ficavam ansiosos quando o dono saía de casa foi significativamente maior $(p<0,05)$ em relação aos que não apresentavam esta alteração. Além disso, foi observada diferença estatisticamente significativa $(p<0,05)$ para os cães e gatos que se tornaram agressivos com outros animais, quando comparados com os que não tiveram tal manifestação.
Para as variáveis "esbarrar em objetos", "agressividade com as pessoas da família", "urinar e defecar dentro de casa", "dificuldade para dormir à noite" e "vocalização excessiva ou inapropriada" não foram detectadas diferenças significativas entre os animais com e sem alterações, o que diferiu de (CHAPMAN e VOITH, 1990). Da mesma forma, não ocorreu diferença estatística entre sexo e mudanças comportamentais, em ambas as espécies, diferente do que foi verificado por BAIN etal. (2001), HART (2001) e NEILSON et al. (2001). Constatou-se que $44,44 \%$ dos gatos idosos apresentaram pelo menos uma mudança senil, ao passo que entre os cães, mais da metade $(53,97 \%)$ tiveram 
algum tipo de alteração comportamental. Isto está de acordo com o relato de que os gatos são capazes de compensar melhor as mudanças senis comparados às outras espécies (CARE, 1983).

Baseando-se nas alterações que podem ocorrer com o envelhecimento, existem atualmente alimentos comerciais balanceados para suprir as necessidades do animal idoso, bem como auxiliares no tratamento de doenças específicas (MARKHAM e HODGKINS, 1989; MOSIER, 1989; SHEFFY et al., 1985; FINCO et al., 1994; TAYLOR et al., 1995). A importância da nutrição em animais idosos reside em diminuir ou prevenir a progressão das mudanças metabólicas, aumentando a expectativa e qualidade de vida (BAILONI e CERCHIARO, 2005).

\section{Conclusões}

Deste estudo retrospectivo foi possível concluir ser a obesidade o distúrbio físico de maior ocorrência em cães e gatos idosos, e a ansiedade e agressividade as mais prevalentes mudanças comportamentais. A combinação de comida caseira com ração comercial é a principal fonte de alimentação oferecida pelos proprietários, sendo a mais consumida pelos animais obesos.

\section{Referências}

BAILONI, L.; CERCHIARO, I. The role of feeding in the maintenance of well-being and health of geriatric dogs. Veterinary Research Communications, v. 29 (suppl. 2), p. 51-55, 2005.

BAIN, M.J.; HART, B.L.; CLIFF, K.D.; RUEHL, W.W. Predicting behavioral changes associated with age-related cognitive impairment in dogs. Journal of the American Veterinary Medical Association, v.218, n.11, p.1792-1795, 2001.

BARR, M.C.; OLSEN, C.W.; SCOTT, F.W. Moléstias virais felinas. In: ETTINGER, S.J.; FELDMAN, E.C. Tratado de medicina interna veterinária. São Paulo: Manole, 1995. cap.70, p.589-631.

BRONSON, R.T. Variation in age at death of dogs of different sexes and breeds. American Journal of Veterinary Research, v.43, n.11, p.2057-2059, 1982.

CARE of old dogs. Feline Practice, v.13, n.5, p.37-40, 1983.

CHAPMAN, B.L.; VOITH, V.L. Behavioral problems in old dogs: 26 cases (1984-1987). Journal of the American Veterinary Medical Association, v.196, n.6, p.944-946, 1990.

CONCANNON, P.W. Endocrinologia reprodutiva, contracepção, e terminação da gestação em cães. In: ETTINGER, S.J.; FELDMAN, E.C. Tratado de medicina interna veterinária. São Paulo: Manole, 1995. cap.124, p.2242-2257.

FINCO. D.R.; BROWN, S.A.; CROWELL, W.A.; BROWN, C.A.; BARSANTI, J.A.; CAREY, D.P.; HIRAKAWA, D.A. Effects of aging and dietary protein intake on uninephrectomized geriatric dogs. American Journal of Veterinary Research, v.55, n.9, p.1282-1289, 1994.
GERMAN, A.J. The growing problem of obesity in dogs and cats. The Journal of Nutrition, v.136 (suppl. 7), p.1940-1946, 2006.

GREELEY, E.H.; BALLAM, J.M.; HARRISON, J.M.; KEALY, R.D.; LAWLER, D.F.; SEGRE, M. The influence of age and gender on the immune system: a longitudinal study in Labrador Retriever dogs. Veterinary Immunology and Immunopathology, v.82, n.1-2, p.5771, 2001.

HART, B.L. Effect of gonadectomy on subsequent development of age-related cognitive impairment in dogs. Journal of the American Veterinary Medical Association, v.219, n.1, p.51-62, 2001.

HOUPT, K.A.; BEAVER, B. Behavioral problems of geriatric dogs and cats. The Veterinary Clinics of North America, Small Animal Practice, v.11, n.4, p.643-652, 1981.

JEUSETTE, I.C.; LHOEST, E.T.; ISTASSE, L.P.; DIEZ, M.O. Influence of obesity on plasma lipid and lipoprotein concentrations in dogs. American Journal of Veterinary Research, v.66, n.1, p.81-86, 2005.

LANDSBERG, G.; ARAUJO, J.A. Behavior problems in geriatric pets. The Veterinary Clinics of North America, Small Animal Practice, v.35, n.3, p.675-698, 2005.

MALINOWSKI, C. Canine and feline nasal neoplasia. Clinical Techniques in Small Animal Practice, v.21, n.2, p.89-94, 2006.

MARKHAM, R.W.; HODGKINS, E.M. Geriatric nutrition. The Veterinary Clinics of North America, Small Animal Practice, v.19, n.1, p.165-185, 1989.

MOSIER, J.E. Effect of aging on body systems of the dog. The Veterinary Clinics of North America, Small Animal Practice, v.19, n.1, p.1-12, 1989.

NEILSON, J.C.; HART, B.L.; CLIFF, K.D.; RUEHL, W.W. Prevalence of behavioral changes associated with agerelated cognitive impairment in dogs. Journal of the American Veterinary Medical Association, v.218, n.11, p.1787-1791, 2001.

PUGLIESE, A.; GRUPPILLO, A.; Di PIETRO, S. Clinical nutrition in gerontology: chronic renal disorders of the dog and cat. Veterinary Research Communication, v.29 (suppl. 2), p.57-63, 2005.

SHEFFY, B.E.; WILLIAMS, A.J. Nutrition and the aging animal. The Veterinary Clinics of North America, Small Animal Practice, v.11, n.4, p.669-675, 1981.

SHEFFY, B.E.; WILLIAMS, A.J.; ZIMMER, J. F.; RYAN, G.D. Nutrition and metabolism of the geriatric dog. Cornell Veterinarian, v.75, p.324-347, 1985.

TAYLOR, E.J.; ADAMS, C.; NEVILLE, R. Some nutritional aspects of ageing in dogs and cats. Proceedings of the Nutrition Society, n.54, p.645-656, 1995.

Recebido para publicação:

$06 / 01 / 2006$ Aprovado: 